\title{
Generating realistic retinal image for whole visual system modeling
}

\author{
Takayuki Kannon*, Shiro Usui \\ From Twentieth Annual Computational Neuroscience Meeting: CNS*2011 \\ Stockholm, Sweden. 23-28 July 2011
}

Recent progress and improvements on optical technology have enabled us to measure the characteristics of the eye in great detail. It has also been shown that, even when myopia and astigmatism are completely corrected with spectacle, the retinal image is still blurred due to uneven refraction power of the cornea and the crystalline lens [1][2], an effect known as "irregular astigmatism". However, almost all researches in visual science assume sharp images as input.

Currently, a large-scale whole visual system models is being developed on a supercomputer to elucidate its complex function [3]. Moreover, it has been demonstrated that the blurred retinal images are gradually compensated at both, the retina and the cortex [4]. Therefore, we have developed an eye optics model which can calculate the blurred retinal image based on known physiological evidences to provide a more realistic input to retinal and visual cortex model.

The proposed model is described by the linear image filter based on Artal's model [5]. It can process multispectral image for reproducing the spectral characteristics of photoreceptors. OTF (Optical transfer function) calculated from the wavefront aberration with spectral transmittance of the lens is used for image processing filter. The wavefront aberration is defined by Zernike Coefficients values (measured by wavefront aberrometer such as OPD-Scan or Shack-Hartman Sensor systems) or SCA values (power of sphero-cylindrical lens with its axis for spectacle lens or contact lens prescriptions). To consider the effects of diffraction, OTF is designed to vary depending on pupil diameter and wavelength. To account for aging effects, the spectral transmittance of the lens [6] was also implemented. As inputs of the

\footnotetext{
* Correspondence: kannon@brain.riken.jp

RIKEN Brain Science Institute, Wako, Saitama, 351-0198, Japan

Full list of author information is available at the end of the article
}

model, multispectral images taken by multispectral camera or converted from RGB image can be utilized.

We simulated a closed-loop system connecting the proposed model to a simple eye movement model and pupil model. The eye movement model generates random saccades and the pupil model modifies pupil diameter according to the mean luminance of retinal image. As a result, our model could reproduce the effect of chromatic aberration, pupil diameter and age on the blurred retinal image. By applying our model to the large-scale visual system model, it is expected to be able to evaluate the phenomena, for example, age dependent perception on visual illusions, blur compensation in retina or visual cortex, and so on.

\section{Published: 18 July 2011}

\section{References}

1. Liang J, Williams DR: Aberrations and retinal image quality of the normal human eye. J Opt Soc Am A Opt Image Sci Vis 1997, 14(11):2873-83.

2. Thibos LN, Hong $X$, Bradley A, Cheng X: Statistical variation of aberration structure and image quality in a normal population of healthy eyes. J Opt Soc Am A Opt Image Sci Vis 2002, 19(12):2329-48.

3. Usui S, Inagaki K, Kannon T, Kamiyama Y, Satoh S, Kamiji NL, Hirata Y, Ishihara A, Shouno H: A Next Generation Modeling Environment PLATO: Platform for Collaborative Brain System Modeling. Neural Information Processing Lecture Notes in Computer Science 2009, 5863/2009:84-90, DOl: 10.1007/978-3-642-10677-4_9.

4. Kannon T, Imazumi Y, Nakauchi S, Sakai H, Usui S: Effect of figure-ground segregation on blur adaptation. Perception 36 ECVP Abstract Supplement 2007.

5. Artal $P$ : Calculations of two-dimensional foveal retinal images in real eyes. J Opt Soc Am A 1990, 7(8):1374-81.

6. van de Kraats J, van Norren D: Optical density of the aging human ocular media in the visible and the UV. J Opt Soc Am A Opt Image Sci Vis 2007. 24(7):1842-57.

doi:10.1186/1471-2202-12-S1-P348

Cite this article as: Kannon and Usui: Generating realistic retinal image for whole visual system modeling. BMC Neuroscience 2011 12(Suppl 1): P348.

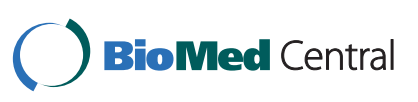

(c) 2011 Kannon and Usui; licensee BioMed Central Ltd. This is an open access article distributed under the terms of the Creative Commons Attribution License (http://creativecommons.org/licenses/by/2.0), which permits unrestricted use, distribution, and reproduction in any medium, provided the original work is properly cited. 